\title{
$\angle S$ Research Square

\section{Impacts of the Marine Hatchery Built Environment on Mucosal Microbiome Colonization Across Ontogeny in Yellowtail Kingfish, Seriola Lalandi}

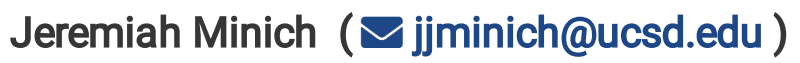

Marine Biology Research Division, Scripps Institution of Oceanography, University of California San

Diego, La Jolla, CA, USA https://orcid.org/0000-0002-7202-965X

Barbara Nowak

University of Tasmania

Abigail Elizur

University Sunshine Coast

Rob Knight

University of California San Diego

Stewart Fielder

NSW DPI: New South Wales Department of Primary Industries

\section{Eric Allen}

University of California San Diego

\section{Research}

Keywords: microbiome, built environment, yellowtail kingfish, Seriola lalandi, aquaculture, fisheries, marine microbiology

Posted Date: September 15th, 2020

DOI: https://doi.org/10.21203/rs.3.rs-75521/v1

License: (c) (1) This work is licensed under a Creative Commons Attribution 4.0 International License. Read Full License 


\section{Abstract}

\section{Background}

Microbial succession in vertebrates has primarily focused on vertical transmission and ontogenetic development in the mammalian gut. Teleosts comprise the majority of vertebrate diversity, yet little is known about how the microbiome develops in fish, particularly when vertical transmission is limited or absent for broadcast spawners. Biological factors such as diet, age, phylogeny, and trophic level along with environmental factors such as water salinity, temperature, and depth have been shown to influence the mucosal microbiomes of fish. Here we investigate how various microbial-rich surfaces from the built environment 'BE' influence the development of the mucosal microbiome (gill, skin, and digesta) of an economically important marine fish, yellowtail kingfish, Seriola lalandi, over time.

Results

For the first experiment, we sampled gill and skin microbiomes from 36 fish reared in three tank conditions, and demonstrate that the gill is more influenced by the surrounding environment than the skin. In a second experiment, fish microbiomes (gill, skin, and digesta) and the BE (tank side, water, inlet pipe, airstones, and air diffusers) were sampled from indoor reared fish at three ages ( $43 \mathrm{dph}, 137 \mathrm{dph}$, $430 \mathrm{dph} ; \mathrm{n}=12$ per age). At $430 \mathrm{dph}, 20$ additional fish were sampled from an outdoor ocean net pen. A total of 304 samples were processed for $16 \mathrm{~S}$ rRNA gene sequencing. Gill and skin alpha diversity increased while gut diversity decreased with age. Diversity was much lower in fish from the ocean net pen compared to indoor fish. We quantified the change in community dynamics driven by the BE and show that the gill and skin are most influenced by the BE early in development, with aeration equipment having more impact in later ages, while the gut microbiome becomes increasingly differentiated from the environment over time.

\section{Conclusions}

Our findings suggest that fish mucosal microbiomes are differentially influenced by the built environment with a high turnover and rapid succession occurring in the gill and skin while the gut microbiome is more stable. We demonstrate how individual components of a hatchery system, especially aeration equipment, may contribute directly to microbiome development in a marine fish. In addition, results demonstrate how early life (larval) exposure to stressors in the rearing environment may influence fish microbiome development which is important for animal health and aquaculture production.

\section{Full Text}

This preprint is available for download as a PDF.

\section{Figures}




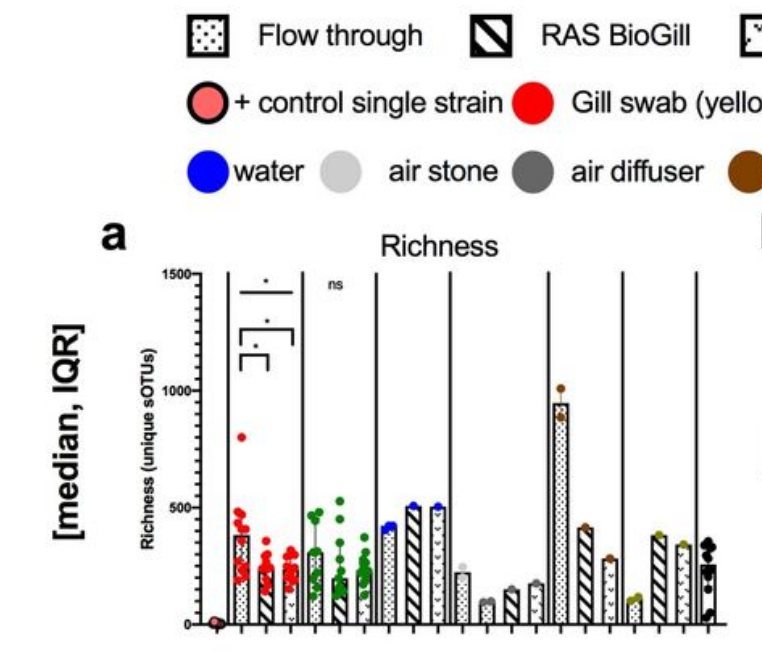

RAS MBBR
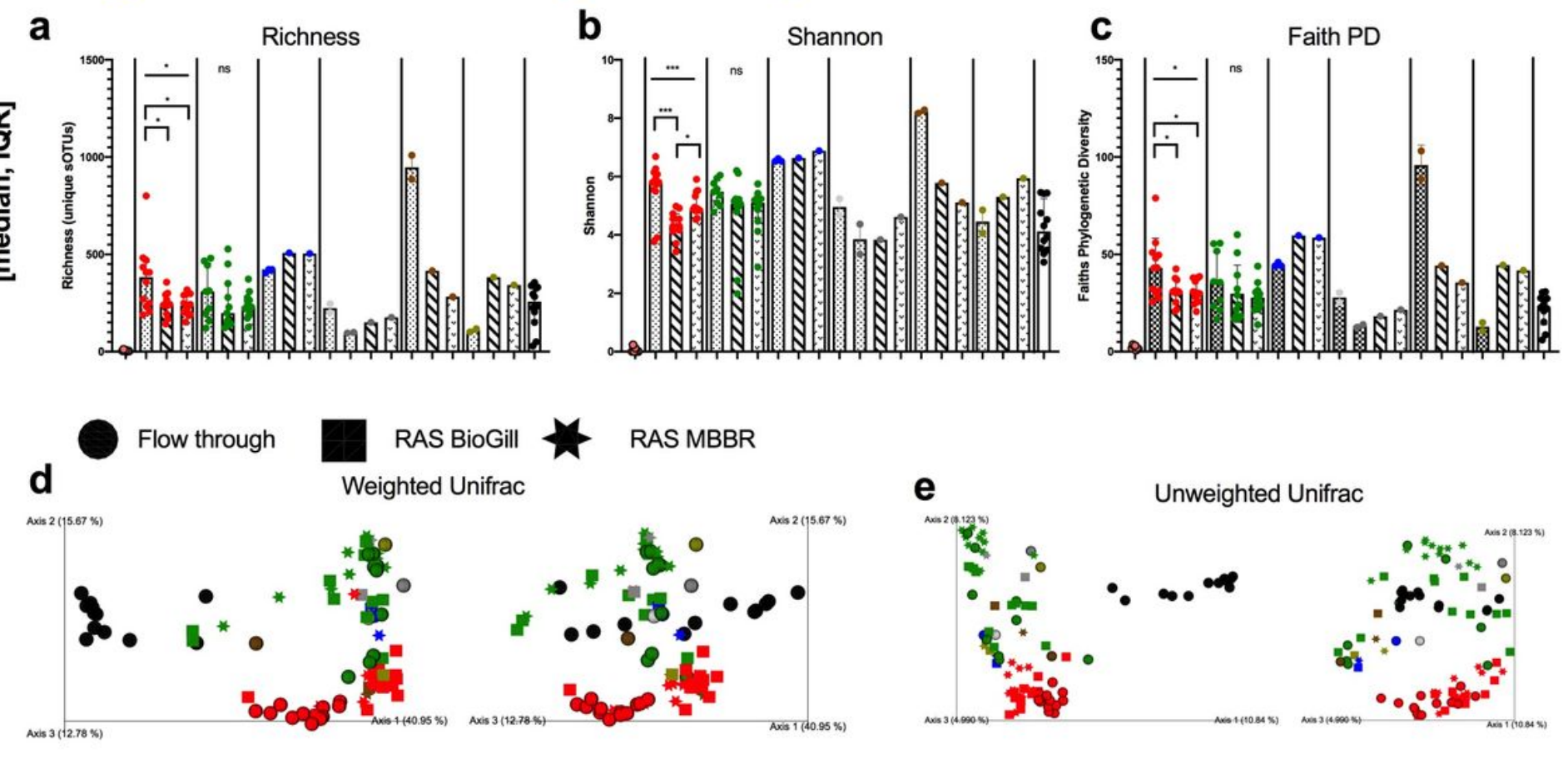

\section{Figure 1}

Microbial diversity of the hatchery built environment along with fish gill and skin mucus at 130 days post hatch across three rearing tanks (flow through, RAS BioGill, and RAS MBBR). Alpha diversity as measured by a) richness, b) Shannon, and c) Faith's phylogenetic diversity. Gill and skin (group comparison calculated with Kruskal-Wallis test, Benjamini Hochberg FDR 0.05). Beta diversity calculated using d)

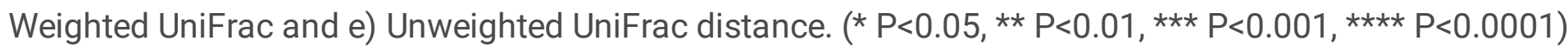



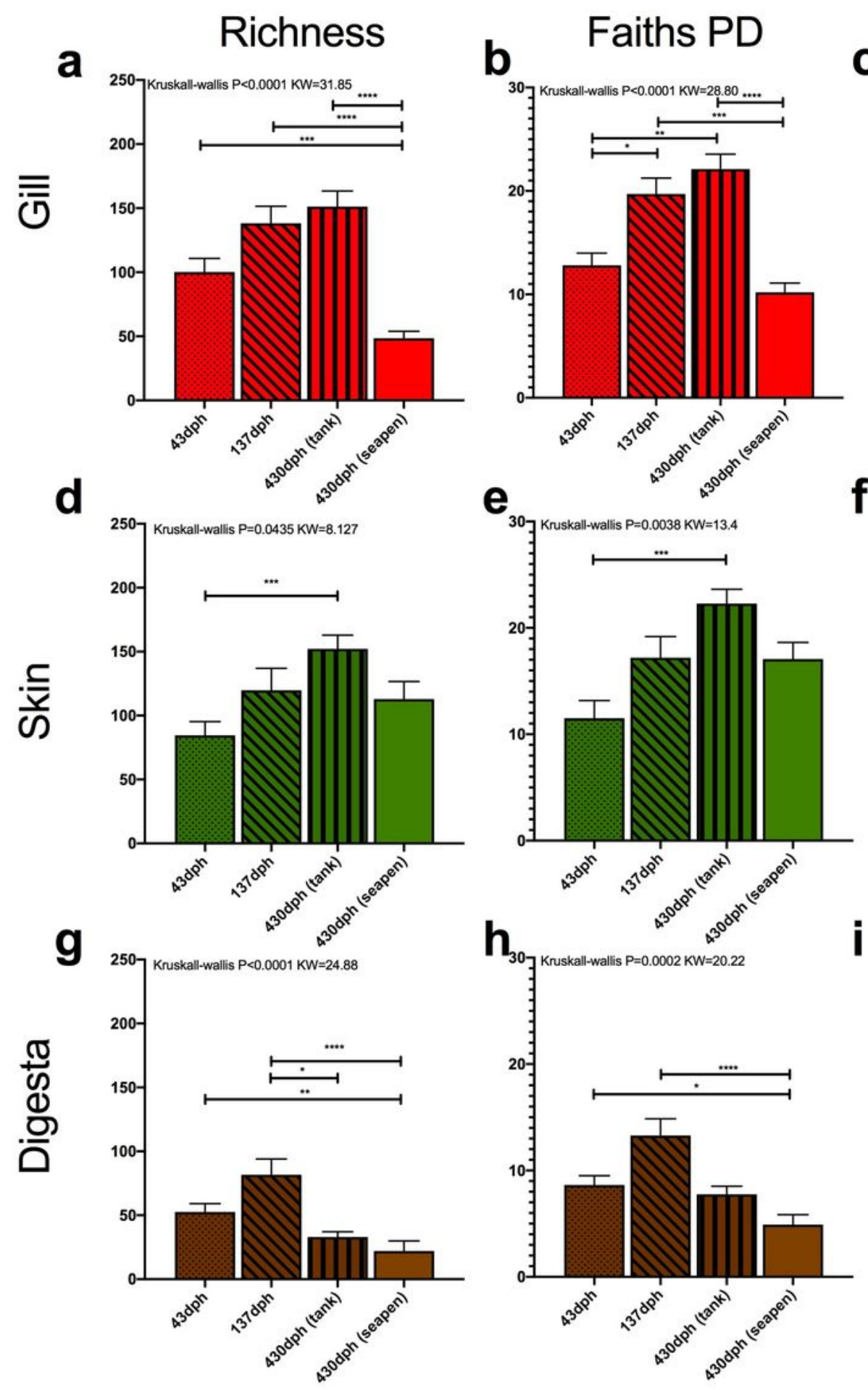

\section{Shannon}

e
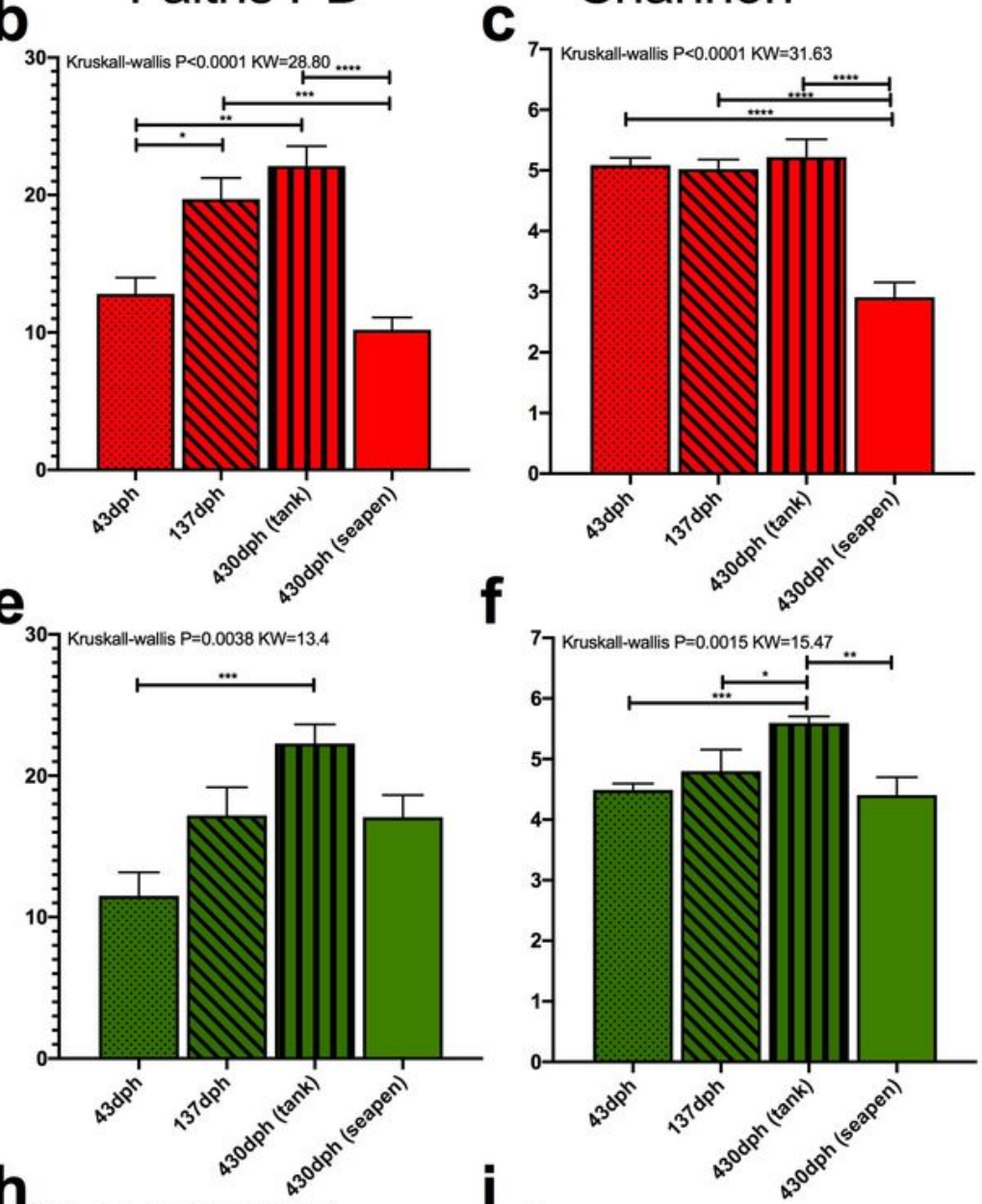

f
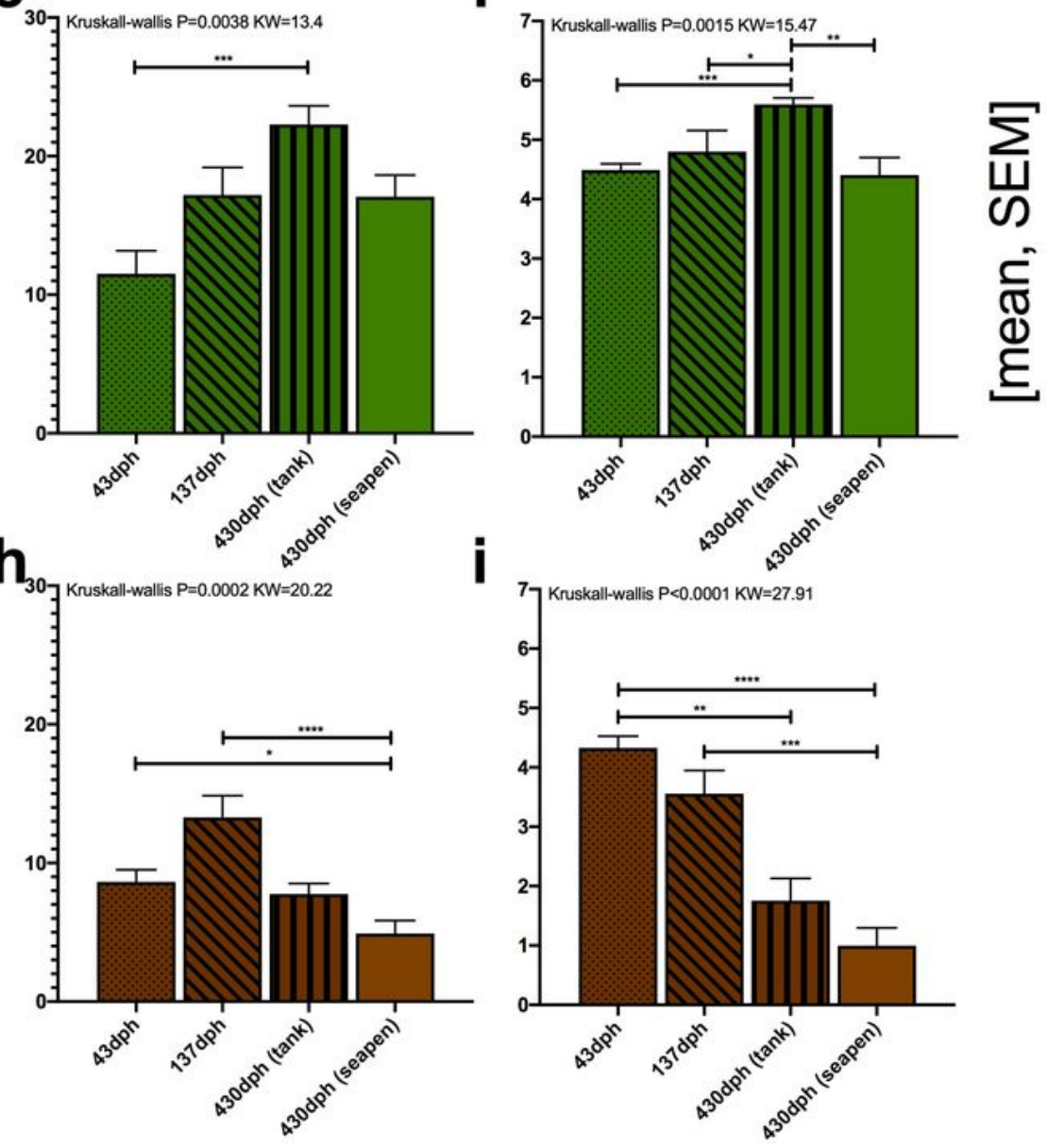

Age (days post hatch)

Figure 2

Alpha diversity measures: richness, Faith's Phylogenetic 934 diversity, and Shannon diversity grouped per body site $($ red = gill, green = skin, brown = digesta). Each body site assessed for diversity differences across age (Kruskal-Wallis, Benjamini-Hochberg FDR 0.05). Gill microbial diversity: a) richness, b) Faiths PD, c) Shannon; Skin microbial diversity: a) richness, b) Faiths PD, c) Shannon; Digesta microbial

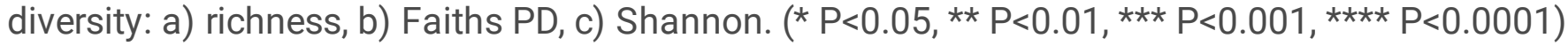



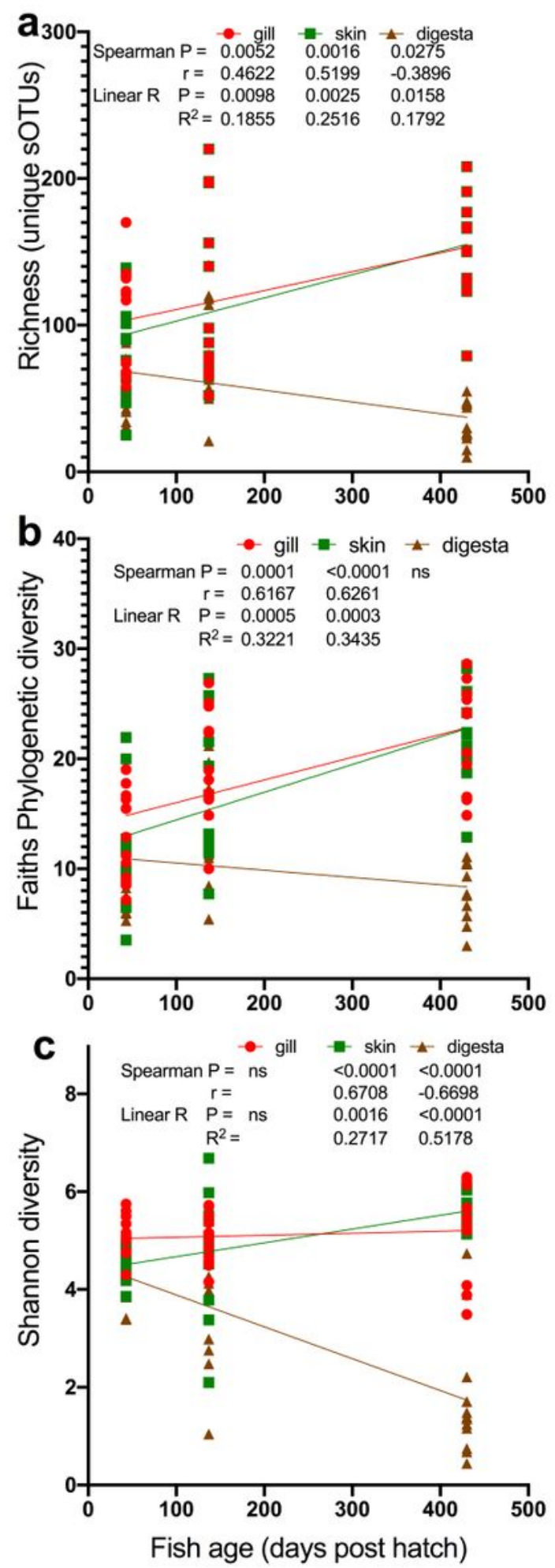

\section{Figure 3}

Modeling of changes in alpha diversity: a) richness, b) Faiths PD, and c) Shannon diversity over the age of the fish. Only fish reared in indoor systems included (430 dph seapen fish excluded). Statistical comparisons of both Spearman correlation and linear model (linear regression) calculated with results depicted on the legends. 

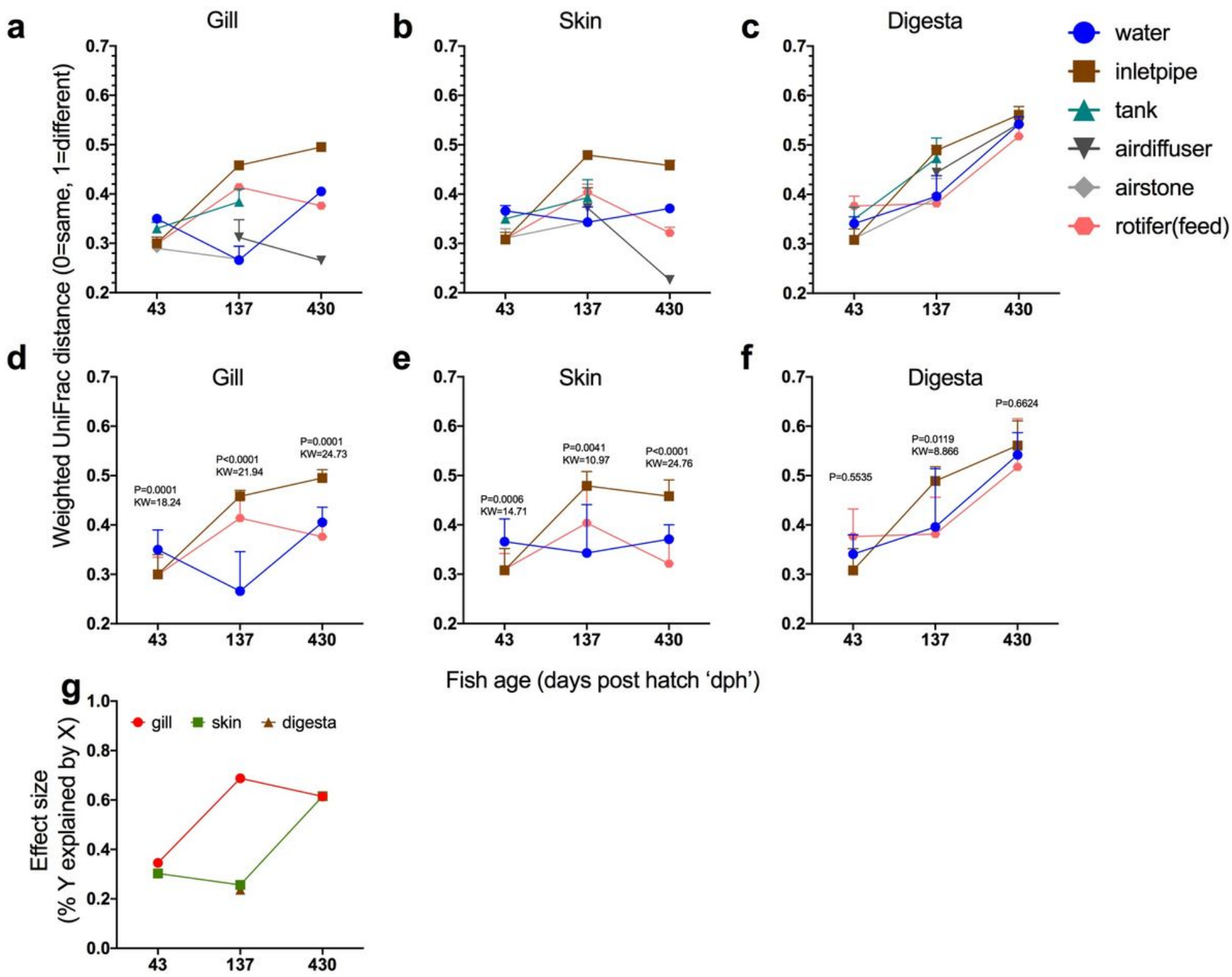

Fish age (days post hatch 'dph')

Fish age (days post hatch 'dph')

\section{Figure 4}

Niche differentiation within body sites over time. Beta diversity distances (weighted normalized UniFrac) of a) gill, b) skin, and c) digesta samples compared to six different hatchery built environment putative microbial sources (water, inlet pipe, tank side, air diffuser, airstone, and first feed (rotifers). Statistical comparison of microbiome differentiation across three BE comparisons (water, inlet pipe, first feed) over time and calculated independently across three body sites: d) gill, e) skin, and f) digesta (Statistical test: Kruskal-Wallis, P value and KW test statistic reported in figure panel. $f$ ) Results from the Kruskal-Wallis test for (d,e,f) depicted as effect size to demonstrate the rate of microbial community niche differentiation. 

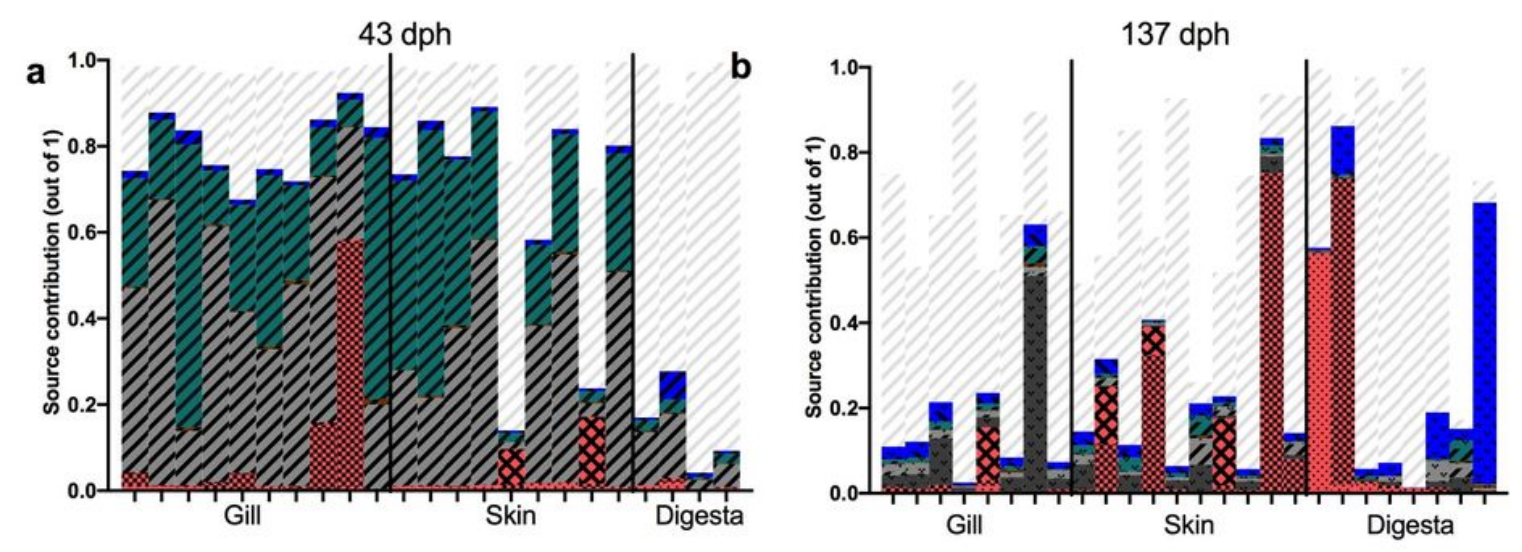

Microbial sources
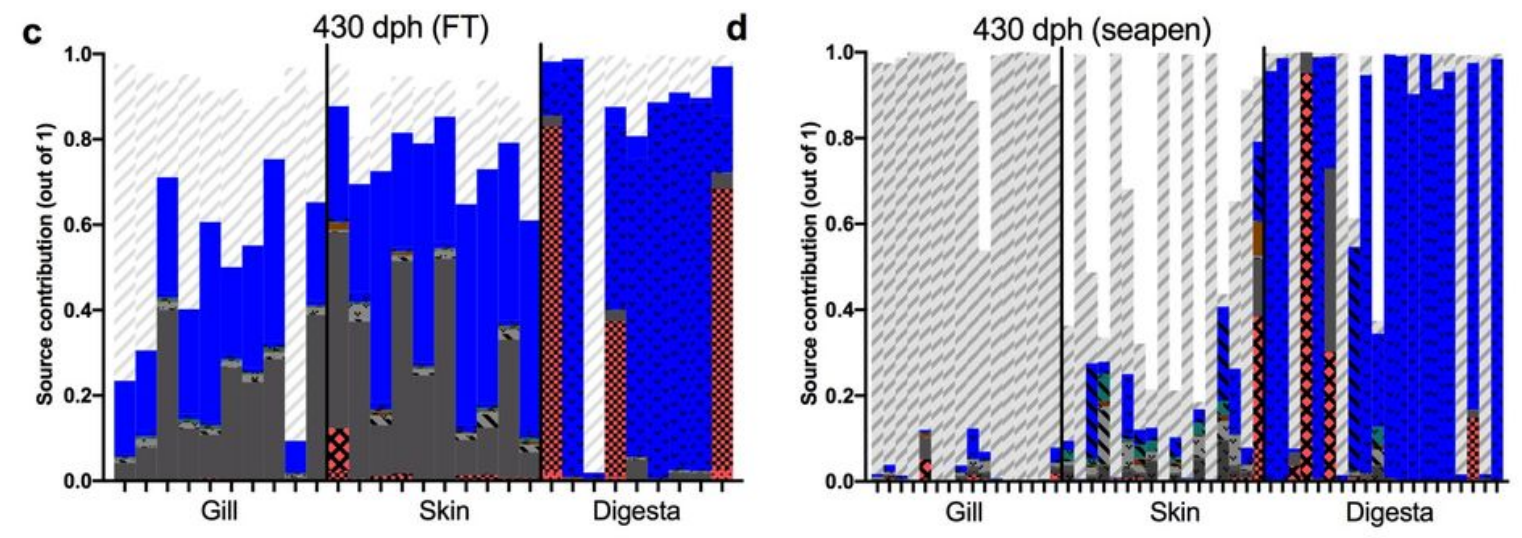

Unknown
Water
430 dph FT
137 dph FT
43 dph
Tank side
137 dph FT
43 dph
Inlet pipe
430 dph FT
137 dph FT
43 dph
Airstone
137 dph FT
43 dph
Air diffuser
430 dph FT
137 dph FT
Feed
Feed (pellet)
Feed (otohime)
Feed-live (rotifer)

\section{Figure 5}

SourceTracker2 analysis of individual microbiome contributions from the built environment onto various mucosal body sites across time: a) $43 \mathrm{dph}$, b) $137 \mathrm{dph}$, c) $430 \mathrm{dph}$ indoor, and d) $430 \mathrm{dph}$ seapen. Features with less than 100 counts across all samples excluded. 'Unknown' indicates source population was not sampled or included thus would be the percentage of a given sample which has source microbes from an unknown location or undetermined source.

\section{Supplementary Files}

This is a list of supplementary files associated with this preprint. Click to download.

- 20200909additionalfilesv1.docx 